\title{
Synthesis and humidity sensing analysis of $\mathrm{ZnS}$ nanowires
}

\author{
Salih Okur ${ }^{\mathrm{a}}$, Neslihan Üzar ${ }^{\mathrm{b}, *}$, Nesli Tekgüzel ${ }^{\mathrm{a}}$, Ayșe Erol ${ }^{\mathrm{b}}$, M. Çetin Arıkan ${ }^{\mathrm{b}}$ \\ a Izmir Institute of Technology, Faculty of Science, Department of Physics Gülbahce Koyu Kampüsü, Urla, 35430 Izmir, Turkey \\ ${ }^{\mathrm{b}}$ Istanbul University, Science Faculty, Physics Department, Vezneciler, 34134 Istanbul, Turkey
}

\section{A R T I C L E I N F O}

\section{Article history:}

Received 6 June 2010

Received in revised form

26 July 2010

Accepted 16 August 2010

Available online 19 August 2010

\begin{abstract}
A B S T R A C T
ZnS nanowires synthesized by the vapor-liquid-solid (VLS) method and humidity sensing properties of obtained ZnS nanowires were investigated by quartz crystal microbalance (QCM) method and electrical measurements. The synthesized nanowires were exposed to relative humidity ( $\mathrm{RH}$ ) between $22 \%$ and 97\% under controlled environment. Our experimental results show that $\mathrm{ZnS}$ nanowires have a great potential for humidity sensing applications in room temperature operations.
\end{abstract}

(c) 2010 Elsevier B.V. All rights reserved.

\section{Introduction}

Semiconductor nanostructures have attracted great attention as materials for sensing gases and humidity due to their superior features such as higher surface-to-volume ratio, lower cost and ease to fabricate as a sensor compared to bulk or thin films [1] and due to high computability with microelectronic processing. Sensing and controlling humidity is very important for many manufacturing environments such as food, automotive, electronics and agriculture industries. Therefore, reliable, cheap, sensitive, low operation temperature and small-sized humidity sensors are indispensible for daily life. Several sensing techniques such as resistance, capacity, optics, field effect transistor (FET), surface acoustic wave (SAW) and QCM can be used to detect humidity [2]. $\mathrm{ZnS}$ is a typical II-VI semiconductor compound with wide direct bandgap energy of $3.68 \mathrm{eV}$ at room temperature and has been widely used for laser, sensor, infrared windows, cathode ray tube and electroluminescence device applications [3,4].

The humidity sensing properties of $\mathrm{ZnS}$ nanowires are not studied using QCM as far as we know. Therefore, here we report the fabrication and characterization of $\mathrm{ZnS}$ nanowires prepared using vapor-liquid-solid (VLS) technique and humidity sensing capabilities using both QCM and electrical measurement techniques.

\section{Experimental}

\subsection{Synthesis of $\mathrm{ZnS}$ nanowires}

The conventional VLS mechanism is based on evaporation of $\mathrm{ZnS}$ powder and transfer of $\mathrm{ZnS}$ vapor to lower temperature regions using an inert gas as a carrier. The growth parameters strongly depend on

\footnotetext{
* Corresponding author. Tel.:+90535427 1271.

E-mail addresses: neslihanuzar@gmail.com, neslihanuzar@yahoo.com (N. Üzar).
}

the source and substrate temperatures, the thickness of catalyst layer and the flow rate of carrier gas. One terminal of the quartz tube was connected to the vacuum pump and the gas flow system was placed in horizontal tube furnace. The quartz boat containing the source material (diameter $10 \mu \mathrm{m}, 99.99 \%$ pure $\mathrm{ZnS}$ powder) and the $\mathrm{Au}$ coated $(50 \AA$ ) Si substrate was placed at the other edge of the quartz tube. Prior to the process, the quartz tube was evacuated by the rotary vacuum pump. After closing the rotary pump, the tube was filled with Ar gas for protecting the process medium from oxygen contamination. When the furnace reached the process temperature $\left(1100{ }^{\circ} \mathrm{C}\right)$, the terminal opposite to the gas flow system was opened and kept open for the gas to flow out during the process. Then the $\mathrm{ZnS}$ powder and the substrate were transferred from the edge of the tube to 1100 and $750{ }^{\circ} \mathrm{C}$ temperature places in the tube, respectively. Evaporated $\mathrm{ZnS}$ vapor was carried by $600 \mathrm{cc} / \mathrm{min}$ of Ar gas and condensed on the substrate. After $1.5 \mathrm{~h}$ process, the quartz boat was taken off rapidly from the quartz tube and the samples were cooled down to room temperature. The Au coated Si substrate was seen to be covered with white wool like layer. Surface morphology, chemical composition and crystal structure of the synthesized structures were examined by SEM, EDS and XRD analyses, respectively.

\subsection{Investigation of humidity sensing capabilities of ZnS nanowires using QCM technique}

QCM has been extensively used to monitor the change in mass loading by measuring the shift of its resonant frequency. The mass change $(\Delta m)$ on surface of the quartz crystal was calculated using Sauerbrey equation [5] from the frequency change $(\Delta f)$ for thin and homogenous film:

$\Delta f=-\frac{2 f_{0}^{2} \Delta m}{A \sqrt{\mu \rho}}$

where $f_{0}$ is the resonant frequency of the fundamental mode of the QCM crystal, $A$ the area of the gold disk coated onto the crystal, $\rho$ the density of the crystal and $\mu$ the shear modulus of quartz. 
QCM with the model of CHI400A series from $\mathrm{CH}$ Instruments (Austin, USA) was used to measure the change in the resonance frequency of quartz crystals via both serial and USB interface connected to a computer. Our QCM apparatus consisted of AT-cut piezoelectric quartz crystal with resonance frequency of $8 \mathrm{MHz}$. The density $(\rho)$ of the crystal was $2.684 \mathrm{~g} / \mathrm{cm}^{3}$ and the shear modulus $(\mu)$ of quartz was $2.947 \times 10^{11} \mathrm{~g} / \mathrm{cm} \mathrm{s}^{2}$. The change of $1 \mathrm{~Hz}$ corresponded to $1.34 \mathrm{ng}$ of materials adsorbed or desorbed onto the crystal surface of an area of $0.196 \mathrm{~cm}^{2}$.

In order to monitor humidity sensing properties, the quartz crystals were cleaned in acetone by ultrasonic waves. Then they were rinsed with deionized water and dried with Ar gas. ZnS nanowires were dispersed in ethanol using ultrasonic cleaner and the solution was applied on the surface of quartz crystal by dropcasting technique. The drop-case solution was dried at room temperature. The thickness of $\mathrm{ZnS}$ nanowires film was measured with a Dektak profilometer from Veeco and found to be around $100 \mathrm{~nm}$. The humidity measurements were taken under various humidity environments of $22-97 \% \mathrm{RH}$ in a closed glass vessel at an ambient temperature of $22{ }^{\circ} \mathrm{C}$. A EI-1050 selectable digital relative humidity and temperature probe with a response time of $4 \mathrm{~s}$ and a resolution of $0.03 \% \mathrm{RH}$ was used with a USB controlled U12 ADC system combined with a single chip sensor module (SHT11) manufactured by Sensirion (Staefa, Switzerland).
2.3. Investigation of humidity sensing capabilities of $\mathrm{ZnS}$ nanowires using electrical measurement techniques

QCM and electrical measurements with respect to relative humidity were carried out using $\mathrm{ZnS}$ nanowires simultaneously in the same environment. The ZnS nanowires-ethanol solution was dropped between the thermally evaporated thickness of $120 \mathrm{~nm}$ gold electrodes with $20 \mu \mathrm{m}$ separation and $1400 \mu \mathrm{m}$ channel width on a glass substrate for electrical resistance measurements. Therefore, ZnS nanowires bridged the two neighboring gold electrodes. Electrical measurements of $\mathrm{ZnS}$ nanowires sensor were measured using a programmable Keithley Sourcemeter, and the commercial humidity/temperature sensor was used to monitor real-time relative humidity changes during the resistance and conductivity measurements.

\section{Results and discussions}

\subsection{Structural characterization of ZnS nanowires}

Fig. 1a-c presents SEM, EDS and XRD images of the ZnS nanowires on $\mathrm{Au}$ coated $\mathrm{Si}$ substrate placed at $750{ }^{\circ} \mathrm{C}$ temperature zone of the horizontal tube. These images show that the lengths of a

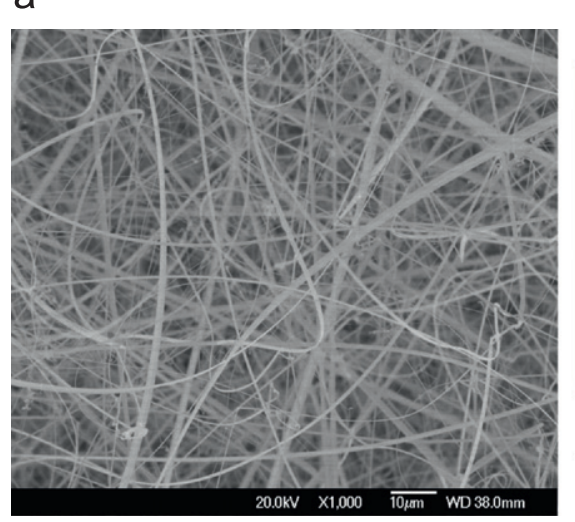

b

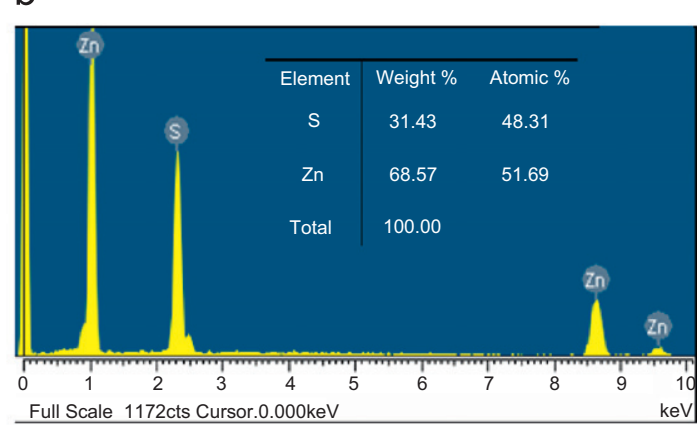

C

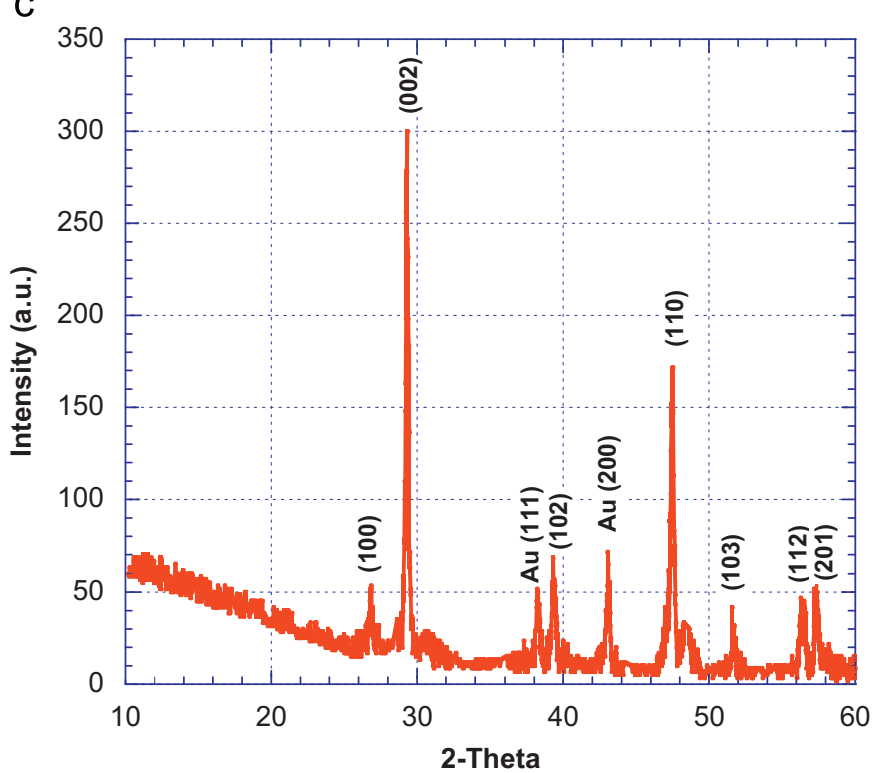

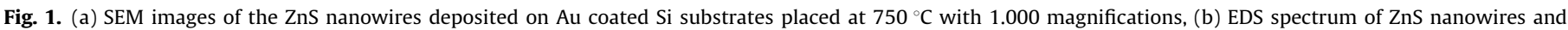
(c) XRD pattern of ZnS nanowires. 
the wurtzite $\mathrm{ZnS}$ nanowires can reach to several tens of micrometers and the diameters of the nanowires change from about 60 to $120 \mathrm{~nm}$. Fig. 1c shows XRD pattern of ZnS nanowires. Diffraction peaks in the XRD pattern are indexed to hexagonal wurtzite-2H structured $\mathrm{ZnS}$ with lattice parameters of $a=3.82098 \mathrm{~nm}$ and $c=6.2573 \mathrm{~nm}$, which matches well with the JCPDS card (36-1450). Also Au diffraction peaks that are seen in XRD spectrum are evident of VLS mechanism.

\subsection{QCM results under varying $R H$}

Fig. 2 shows the frequency shift of $\mathrm{ZnS}$ nanowires sensor as a function of time for different relative humidities. This works with a humidity cycle of low (22\% RH)-to-high (97\% RH) and high (97\% RH)-to-low (22\%RH) step. It can be seen from the figure that the differences between the ascending and descending curves are small, indicating the sensor has good frequency reproducibility and low hysteresis.

Fig. 3 shows that change in the frequency shift of $\mathrm{ZnS}$ nanowires thin film coated quartz crystal was varied cyclically as a function of time for different relative humidities within a time interval of $200 \mathrm{~s}$. The experiment was repeated at room temperature at two relative humidity points: $45 \% \mathrm{RH}$ and about 75\% RH. While the red line was indicated by the QCM result, results shown in blue line were obtained by commercial humidity

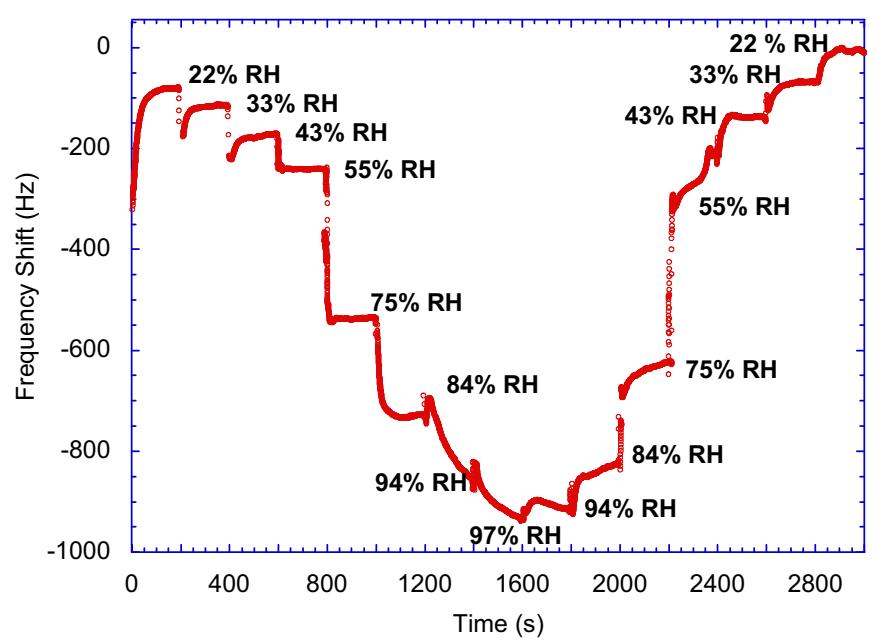

Fig. 2. Frequency shift as a function of time for different RH levels step by step.

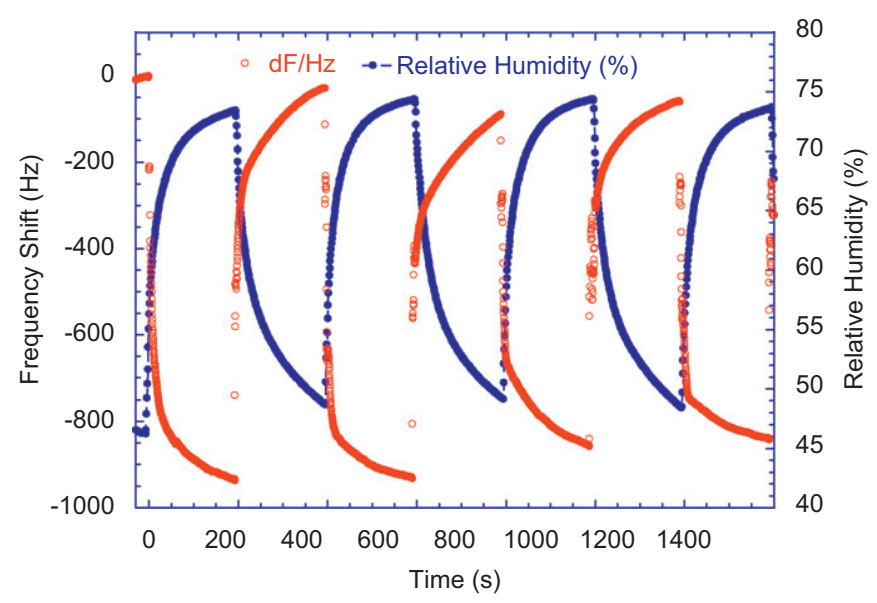

Fig. 3. Repeatable responses of $\mathrm{ZnS}$ nanowire sensor during four cycles between $45 \%$ and about $75 \% \mathrm{RH}$. sensor. The oscillating resonance frequency of QCM electrodes decreased sharply with an increase in RH, i.e. frequency shift of ZnS sensor increased at negative values corresponding to Sauerbrey equation. On the other hand, during the desorption process, the $\mathrm{RH}$ was decreased suddenly and then QCM recovered back to its initial resonance frequency value due to desorption of moisture molecules from the drop-casted $\mathrm{ZnS}$ nanowires surface on the electrodes. The magnitude of change in the oscillating frequency was lower in the last two adsorptiondesorption cycles compared to the other repeated cycles due to slower adsorption rate.

Fig. 4 shows the relationship between the frequency shift and the corresponding relative humidity for $\mathrm{ZnS}$ nanowires sensor. The maximum frequency shifts of the samples to the relative humidity from $22 \%$ to $97 \%$ seemed all around $1000 \mathrm{~Hz}$. It was clear that when the relative humidity was increased, the amount of adsorbed moisture molecules also increased on the surface of $\mathrm{ZnS}$ nanowires. The moisture molecules are usually thought to be physically adsorbed on the surface of $\mathrm{ZnS}$ nanowires and located in spaces of structure. This is why $\mathrm{ZnS}$ nanowires posses a large specific surface area, more rough surface moisture and spaces between the treated nanowires became larger, molecules adsorb easily on the sensor surface and the mass of quartz crystal increases. It is clear that the $\mathrm{ZnS}$ nanowires are very sensitive to the change in relative humidity having a fast response and the interaction with humidity molecules and $\mathrm{ZnS}$ nanowires is nearly reversible.

\subsection{Electrical measurement results under varying $R H$}

Fig. 5 shows the response of $\mathrm{ZnS}$ nanowires sensor versus time for repetitive pulse of $45 \%$ and $75 \% \mathrm{RH}$ at $5 \mathrm{~V}$. The response of $\mathrm{ZnS}$ nanowires against \%RH is defined as $R_{\mathrm{air}} / R_{\mathrm{RH}}$. The response of $\mathrm{ZnS}$ nanowires increases with increase in \%RH depending on decreasing the resistance of $\mathrm{ZnS}$ nanowires, which changed from $2 \times 10^{10}$ to $6 \times 10^{6} \Omega$ at $45 \%$ and $75 \% \mathrm{RH}$, respectively.

The relationship between the variations in response of $\mathrm{ZnS}$ nanowires and the RH variation of $22-97 \%$ was shown in Fig. 6. The response of $\mathrm{ZnS}$ nanowires increases almost exponentially with an increase in $\mathrm{RH}$. This increase in response is about four orders. This result showed that $\mathrm{ZnS}$ nanowires are good resistive

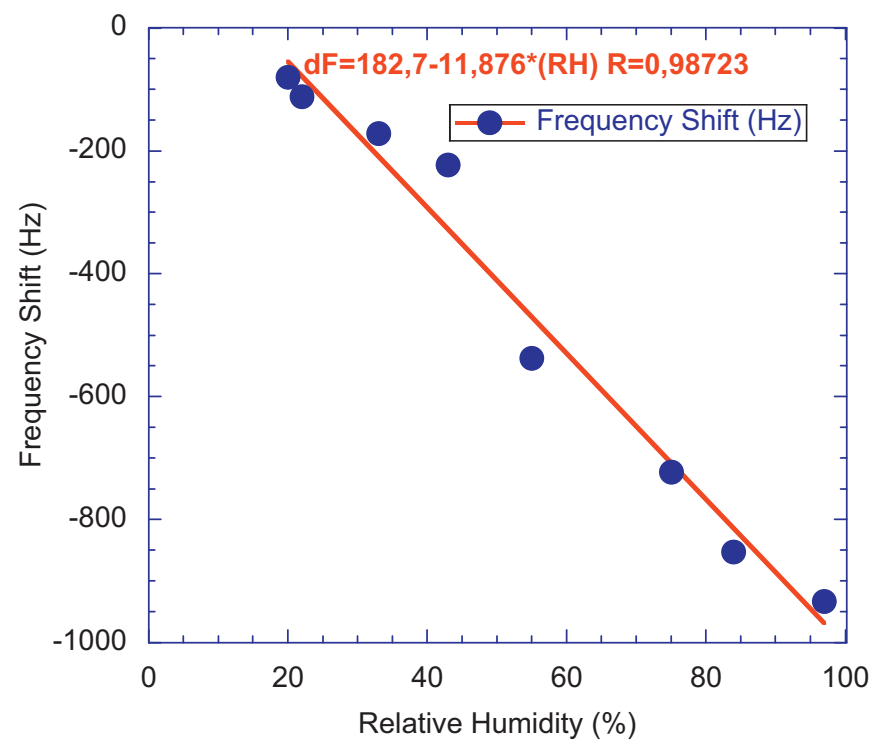

Fig. 4. Relationship between the frequency shifts of ZnS nanowire coated QCM crystal and different relative humidities. 


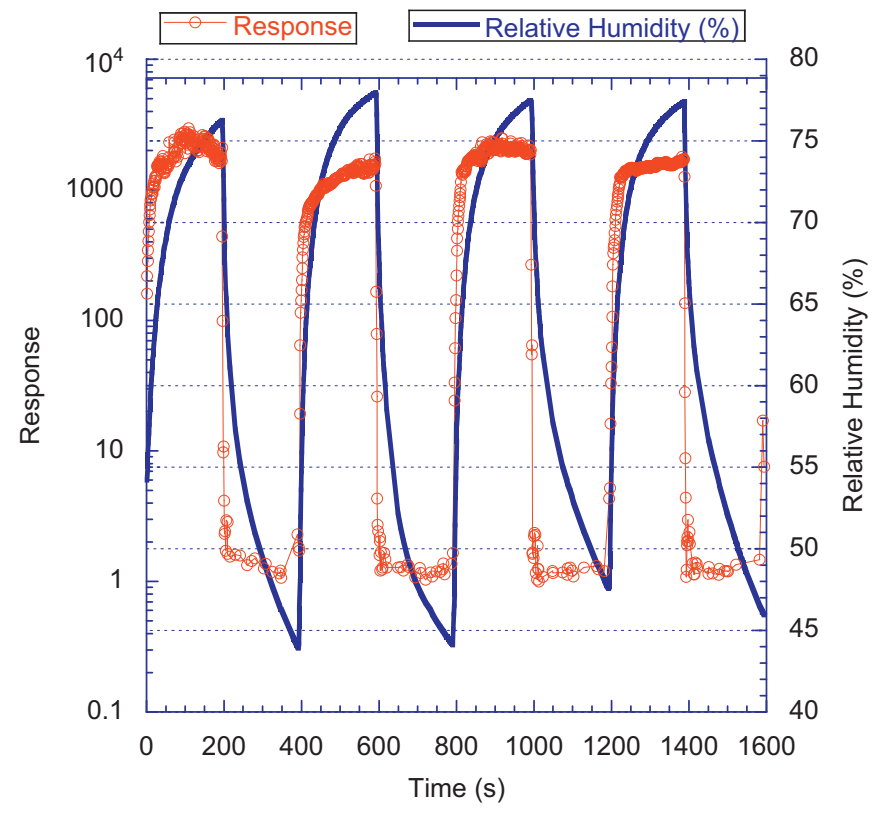

Fig. 5. Variation in response values of $\mathrm{ZnS}$ nanowire sensor (circles line) and humidity levels measured by a commercial humidity sensor (squares line) at $45 \%$ and $75 \% \mathrm{RH}$.

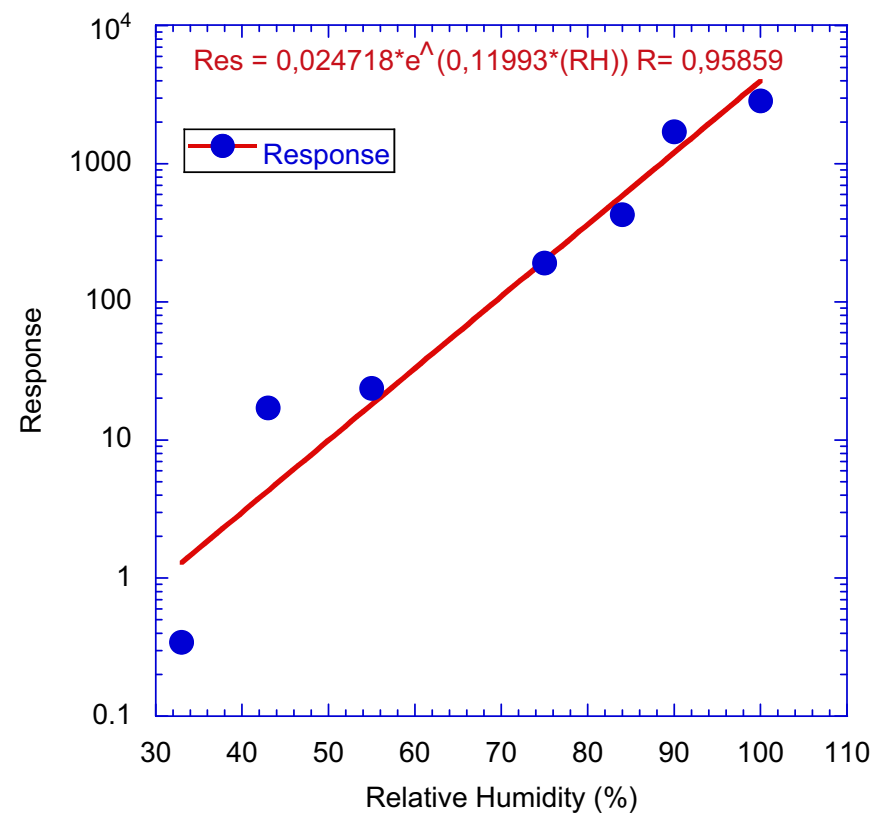

Fig. 6. Variation in sensor values of $\mathrm{ZnS}$ nanowires versus $22 \%, 33 \%, 43 \%, 55 \%, 75 \%$, $84 \%$ and $97 \% \mathrm{RH}$.

type humidity sensors. The resistive type semiconductor sensor mechanism can be understood as follows: adsorption of the moisture affects the protonic conduction on the sensor surface and resistance varies the amount of water adsorbed by it [6]. Reversible physisorption of water molecules is a strong function of humidity. A decrease in resistance can occur only through the water layers by the electrical migration of either protons or hydroxyl ions. A single monolayer of chemisorbed hydroxyl groups can conduct by proton hopping due to hydroxyl dissociation under the influence of an applied field. If physisorbed water is present then hydroxyl ions can diffuse to the defects in lattice.
Physisorbed water is ionized and the extra proton hops from molecule to molecule by a process called the Grotthuss chain reaction. The other idea would be the capillary condensation. For randomly grown nanowires, one can treat the numerous and irregular voids in between the nanowires as interconnecting capillary pores, which increase the surface areas for the adsorption of water molecules [7].

Typical current-voltage $(I-V)$ curves of $\mathrm{ZnS}$ nanowires sensor from $22 \% \mathrm{RH}$ to $97 \% \mathrm{RH}$ are shown in Fig. 7. Conductivity of $\mathrm{ZnS}$ nanowires sensor increasing with an increase in relative humidity is related to the amount of the absorbed moisture molecules or atoms on the surface of $\mathrm{ZnS}$ nanowires sensor. Thus, the amount of dope atoms increases in the $\mathrm{ZnS}$ semiconductor nanowires and decreases the resistance value of $\mathrm{ZnS}$ nanowires sensor. The current-voltage curves are a straight line, showing an ohmic behavior. This suggests that the conductivity in the system is mainly electronic [8].

There are studies about humidity sensing properties of similar nanomaterials, for example $\mathrm{ZnO}$ using $\mathrm{QCM}$ and resistance measurement techniques. The experimental results show that the resistance of $\mathrm{ZnO}$ nanowires changes by about four orders of magnitude [9] when exposed to moisture of $97 \%$ relative humidity and the frequency shift of $\mathrm{ZnO}$ coated QCM crystal is about $900 \mathrm{~Hz}$ at $80 \% \mathrm{RH}$ [10]. If we compare our results with these results, we can say that $\mathrm{ZnS}$ nanowires exhibit similar sensing properties with $\mathrm{ZnO}$ nanostructures.

\subsection{Theoretical analysis of QCM results using modified Langmuir model}

To describe the adsorption kinetics of gas molecules onto organic or inorganic films, the Langmuir adsorption isotherm model is frequently used [11-14]. The rate of surface reaction to form a monolayer on the surface is described by this model using the following equation:

$\frac{d \theta}{d t}=k_{a}(1-\theta) C-k_{d} \theta$

where $\theta$ is the fraction of surface coverage, which is a unitless quantity, $C$ the concentration of the gas in the air, $k_{a}$ the adsorption rate constant and $k_{d}$ the desorption rate constant. Integration of Eq. (2) leads to

$\theta(t)=K^{\prime}\left(1-e^{-k_{o b s} t}\right)$

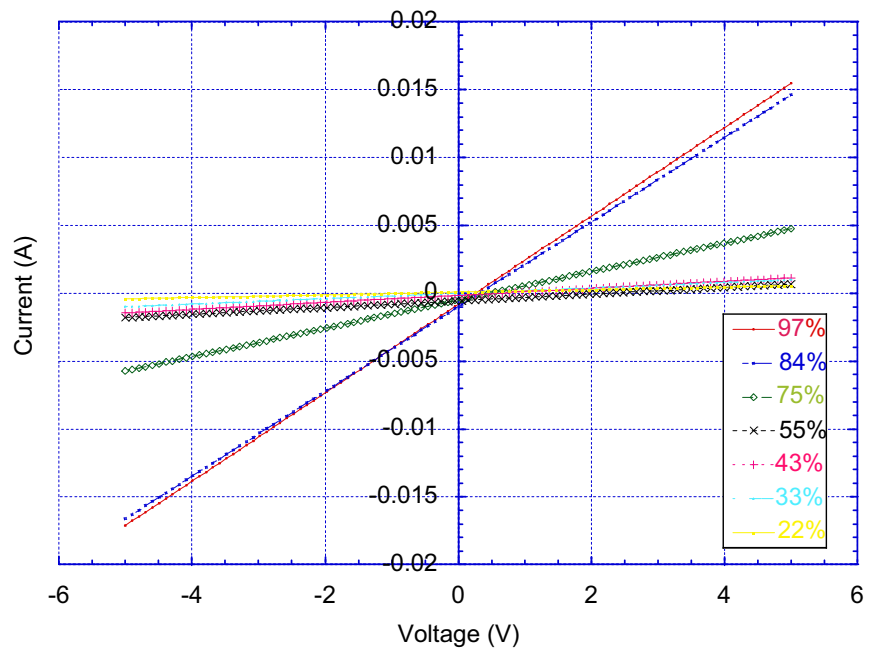

Fig. 7. $I-V$ characterization of $\mathrm{ZnS}$ nanowire sensor from $22 \%$ to $97 \% \mathrm{RH}$. 


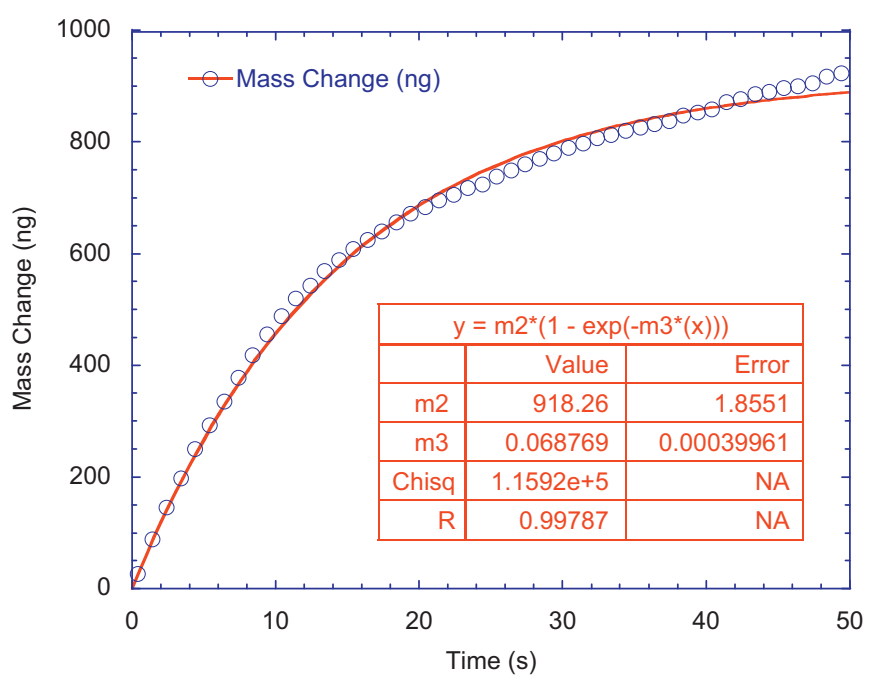

Fig. 8. Least square fit of the adsorption part of the second cycle given in Fig. 3 to the Langmuir adsorption isotherm model.

where $K^{\prime}$ is the association constant and $k_{o b s}$ the inverse of the relation time defined as

$K^{\prime}=\frac{C}{C+k_{d} / k_{a}}$ and $k_{o b s}=k_{a} C+k_{d}$

In the QCM technique, increase in the frequency shift is proportional to the mass uptake of moisture molecules. The Langmuir isotherm adsorption can fit the time dependent frequency shift. In Fig. 8, the least square fit of the adsorption part given in Fig. 3 is shown. Association constant $\left(K^{\prime}\right)$ of the water vapor molecules is found as $918.26 \pm 1.85$, and $k_{o b s}=0.068769 \pm$ $0,0004 \mathrm{~s}^{-1}$ from the curve fit.

Using the Sauerbrey relation given in Eq. (1), ( $\Delta m=-(1.34 \mathrm{ng} /$ $\mathrm{Hz}) \Delta f$, the time dependent variation in mass of the adsorbed water vapor molecules on the $\mathrm{ZnS}$ nanowire film surface $\Delta m_{t}$ can be obtained as follows:

$\Delta m_{t}=\Delta m_{\infty}\left(1-e^{-t / \tau}\right)$

$\tau^{-1}=k_{a}[$ water vapour molecules $]+k_{d}$

$\Delta m_{\infty}$ is the maximum adsorbed amount of the humidity molecules on the surface when $t \rightarrow \infty$ and $\tau$ the relaxation time. From the least square fit to Eq. (5), the relaxation time of the adsorption process is calculated as $42.259 \mathrm{~s}$.

The adsorption and the desorption rate values were obtained to be $55.962 \mathrm{M}^{-1} \mathrm{~s}^{-1}$ and $0.023 \mathrm{~s}^{-1}$, respectively, from the least square fit of the adsorption part of one of the cycle.

From the fit results, the equilibrium constant, $K_{\text {equ }}$, for $\mathrm{ZnS}$ nanowire film can be determined using the following relation:

$K_{\text {equ }}=k_{a} / k_{d}$

The Gibbs free energy $\Delta G$ of adsorption and desorption process in terms of $K_{\text {equ }}$ at a given constant temperature is defined as [15]

$\Delta G=-R T \ln K_{\text {equ }}$

Gibbs free energies for adsorption processes are calculated using Eq. (8) for second cycle. The $K_{\text {equ }}$ and Gibbs free energy for adsorption in the second cycle are obtained as $2365 \mathrm{~s}^{-1}$ and $-7.769 \mathrm{~kJ} / \mathrm{mol}$, respectively. Because of the condensation of water molecules on ZnS nanowire film surface, there is an energy loss that is shown with the negative sign.

The QCM results show that ZnS nanowire films are extremely sensitive to humidity changes at room temperature and give reproducible adsorption kinetics against humidity changes for short time intervals. Hence, $\mathrm{ZnS}$ nanowires have high potential to react with water molecules, i.e. $\mathrm{ZnS}$ nanowires are sensitive to water molecules. Our experimental results also show that the $\mathrm{ZnS}$ nanowire films are quite stable for humidity changes during measurements.

\section{Conclusion}

ZnS nanowires film on electrodes of $100 \mathrm{~nm}$ thickness was obtained by spin coating technique. QCM and electrical responses of $\mathrm{ZnS}$ nanowires synthesized by VLS method against relative humidity were investigated. The adsorption kinetics under constant relative humidity between $45 \%$ and $75 \%$ was analyzed using the Langmuir absorption model at $22{ }^{\circ} \mathrm{C}$ constant QCM temperature. Of QCM and electrical method, electrical method has more sensitivity and stability for sensing humidity based on $\mathrm{ZnS}$ nanowires, because they exhibit high sensitivity for the humidity, showing nearly four order changes in the resistance between $22 \%$ and $97 \% \mathrm{RH}$. This is why $\mathrm{ZnS}$ nanowires sensor can be used as resistive humidity sensors. Conductivity of $\mathrm{ZnS}$ nanowires sensor increases with an increase in relative humidity. The values of adsorption rate, desorption rate and equilibrium constant were obtained as $55.962 \mathrm{M}^{-1} \mathrm{~s}^{-1}, 0.023 \mathrm{~s}^{-1}$ and 2365 , respectively. The Gibbs free energy for adsorption cycle is obtained as $-7.769 \mathrm{~kJ} / \mathrm{mol}$. A ZnS nanowires-based sensor was fabricated and sensing characteristics to relative humidity were studied. QCM and its sensitivity results exhibit that for fabricating highly sensitive humidity sensors, the $\mathrm{ZnS}$ nanowires can be used.

\section{Acknowledgements}

This research was partially supported by DPT (State Planning Organization of Turkey) under Project number DPT2003K120390, by Tubitak (Turkish Scientific Association) under Project number TBAG $109 \mathrm{~T} 240$ and by the Research Fund of the Istanbul University in Turkey with Project number T-676.

\section{References}

[1] J. Riu, A. Maroto, F.X. Rius, Talanta 69 (2006) 288.

[2] H.W. Chen, R.J. Wu, K.H. Chan, Y.L. Sun, P.G. Su, Sensors and Actuators B 104 (2005) 80.

[3] M. Bredol, J. Merichi, Journal of Materials Science 33 (1998) 471.

[4] P. Calandra, M. Goffredi, V.T. Liveri, Colloids Surface A 160 (1999) 9.

[5] G. Sauerbrey, Zeitschrift für Physik 155 (1959) 206

[6] B.C. Yadav, R. Srivastava, C.D. Dwivedi, P. Pramanik, Sensors and Actuators A 153 (2009) 137.

[7] Y.P. Leung, W.C.H. Choy, T.I. Yuk, Chemical Physics Letters 457 (2008) 198.

[8] Y. Li, M.J. Yang, G. Casalbore-Miceli, N. Camaioni, Synthetic Metals 128 (2002) 293.

[9] Y. Zhang, K. Yu, D. Jiang, Z. Zhu, H. Geng, L. Luo, Applied Surface Science 242 (2005) 212.

[10] X. Zhou, J. Zhang, T. Jiang, X. Wang, Z. Zhu, Humidity 135 (2007) 209.

[11] D.S. Karpovich, G.J. Blanchard, Langmuir 10 (1994) 3315.

[12] S.J. Gregg, K.S.W. Sing, in: Adsorption, Surface Area and Porosity, Academic Press, London, ISBN 0-12-300956-1, 1967.

[13] Y.L. Sun, R.J. Wu, Y.C. Huang, P.G. Su, M. Chavali, Y.Z. Chen, C.C. Lin, Talanta 73 (2007) 857.

[14] P.G. Su, Y.P. Chang, Sensors and Actuators B 129 (2008) 915.

[15] S. Qiu, L. Sun, H. Chu, Y. Zou, F. Xu, N. Matsuda, Thin Solid Films 517/9 (2009) 2905. 